\title{
Approach to a Child with Irritable Bowel Syndrome: A Review
}

\author{
Md. Rukunuzzaman'1, ASM Bazlul Karim², Wahiduzzaman Mazumder³, Faika \\ Hussain $^{4}$, Mohammed Nurullah ${ }^{5}$, Kaniz Sultana ${ }^{6}$ \\ ${ }^{1}$ Associate Professor, Department of Paediatric Gastroenterology, Banghabandhu Sheikh Mujib Medical \\ University, Dhaka, Bangladesh; ${ }^{2}$ Professor \& Chairman, Department of Pediatric Gastroenterology and \\ Nutrition, Banghabandhu Sheikh Mujib Medical University, Dhaka, Bangladesh; ${ }^{3}$ Assistant \\ Professor, Department of Paediatric Gastroenterology, Banghabandhu Sheikh Mujib Medical \\ University, Dhaka, Bangladesh; ${ }^{4}$ Registrar, Department of Paediatrics, Shaheed \\ Suhrawardy Medical College Hospital, Dhaka, Bangladesh; ${ }^{5}$ Resident Pediatric \\ Gastroenterology and Nutrition, Banghabandhu Sheikh Mujib Medical \\ University, Dhaka, Bangladesh; ${ }^{6}$ Outdoor Medical Officer Eye, \\ Dhaka Medical College Hospital, Dhaka, Bangladesh
}

[Received: January 2015; Revised: March 2015; Accepted: June 2015; Published: January 2016]

\begin{abstract}
Irritable bowel syndrome (IBS) is one of the most common and best studied disorders among the group of functional gastrointestinal disorders. It is a functional bowel disorder in which abdominal pain or discomfort is associated with defecation or a change in bowel habit. Visceral hypersensitivity and increased GIT motility are the main patho-physiological mechanism for developing IBS. IBS present with diarrhoea or constipation or both. Investigations are least needed for diagnosis of IBS rather done to exclude differential diagnosis. Diagnosis of IBS is done on the basis of Rome-III criteria. Proper counseling, dietary management, antispasmotic and antidepressant are the mainstay of treatment. [Journal of National Institute of Neurosciences Bangladesh, 2016;2(1): 34-39]
\end{abstract}

Keywords: Abdominal pain, children, irritable bowel syndrome

Correspondence: Dr. Md. Rukunuzzaman, Associate Professor, Department of Paediatric Gastroenterology, Banghabandhu Sheikh Mujib Medical University, Dhaka, Bangladesh; Email : dr.rukon@gmail.com; Cell no: +880176835025

Conflict of interest: There was no conflict of interest among the authors.

Contribution of authors: All the authors revised the manuscript and participated in language editing.

How to cite this article: Rukunuzzaman M, Karim ASMB, Mazumder W, Hussain F, Nurullah M, Sultana K. Approach to a Child with Irritable Bowel Syndrome: A Review. J Natl Inst Neurosci Bangladesh, 2016;2(1): 34-39

Copyright: (C2016 Rukunuzzaman et al. Published by Journal of National Institute of Neurosciences Bangladesh. This article is published under the Creative Commons CC BY-NC License (https://creativecommons.org/licenses/by-nc/4.0/). This license permits use, distribution and reproduction in any medium, provided the original work is properly cited, and is not used for commercial purposes.

\section{Introduction}

Irritable bowel syndrome (IBS) has been recognized as one of the most common and best studied disorder among the group of functional gastrointestinal disorders $^{1}$. It is a functional bowel disorder in which abdominal pain or discomfort is associated with defecation or a change in bowel habit ${ }^{2}$. Prevalence of IBS in adults is gradually increasing, about $20 \%$ in western population ${ }^{3}, 6.5-10.1 \%$ in Asian countries ${ }^{4}$ and $4 \%$ in northern India, showing in recent studies 5 . One study of children in North America found that $14 \%$ of high school students and $6 \%$ of middle school students have IBS. The study also found that IBS affects boys and girls equally ${ }^{6}$. Through its exact incidence is hard to assess, but it appears that those attending gastroenterology clinics represent a few person go to the tertiary centre from where the above prevalence data are collected ${ }^{7}$.

Some questions therefore remain to be answered - Is there a significant difference in the incidence of IBS in developing and developed countries? Will the incidence of IBS increase by adoption of a Western lifestyle? Are predisposing factors different in developing and developed countries? Is the increasing stress on young children to achieve or family break-up responsible for IBS symptoms becoming evident at a younger age? The answer is probably 'yes' to a certain extent. Infective pathology is considered the trigger for IBS in developing country. According to Rome III criteria1, for the diagnosis of IBS following requirements are 
needed- Criteria must include both of the following:

1. Abdominal discomfort or pain associated with two or more of the following at least $25 \%$ of the time: a) Improvement with defecation; b) Onset associated with a change in frequency of stool and c) Onset associated with a change in form (appearance) of stool

2. No evidence of an inflammatory, anatomic, metabolic, or neoplastic process that explains the subject's symptoms. Criteria fulfilled at least once per week for at least 2 months prior to diagnosis. Discomfort means an uncomfortable sensation not described as pain.

\subsection{Pathophysiology of IBS}

Pathophysiology of IBS is multifactorial.

2.1. Visceral Hypersensitivity: Increased sensitivity to stimuli is a frequent finding in irritable bowel syndrome patients ${ }^{8}$. Perception in the gastrointestinal tract results from stimulation of various receptors in the gut wall. These receptors transmit signals via afferent neural pathways to the dorsal horn of the spinal cord and ultimately to the brain. Hyper-sensitization of visceral afferent nerves in the gut, triggered by bowel distention or bloating may be the possible explanation for IBS. Hypersensitivity of the intestines to normal sensations may be mediated by the local GI nervous system, by central modulation from the brain, or by some combination of the two9. Other factors may contribute to visceral hyperalgesia, such as specific gastrointestinal mediators (serotonin, kinins) ${ }^{10}$.

2.2. Gastrointestinal Motility: Motor abnormalities of the gastrointestinal tract are detectable in some patients with IBS. Abnormalities include increased frequency and irregularity of luminal contractions, prolonged transit time in constipation-predominant IBS, and an exaggerated motor response to cholecystokinin and meal ingestion in diarrhea-predominant IBS ${ }^{11}$.

2.3. Intestinal Inflammation- Mucosal immune system activation occurs and is characterized by alterations in particular immune cells and markers in some patients with irritable bowel syndrome. Increased numbers of lymphocytes and mast cells are found in the colon and small intestine in patients with IBS $^{12,13}$. Elevated levels of plasma pro-inflammatory interleukins have been observed in patients with IBS. In addition, peripheral blood mononuclear cells of IBS patients produce higher amounts of tumor necrosis factor than healthy controls

2.4. Post-Infectious: Irritable bowel syndrome may occur following infectious enteritis. The increased risk of post-infectious IBD is associated with bacterial, protozoan, helminthes, infections, and viral infections. The cause of bowel symptoms following acute infection is uncertain, although several theories have been proposed $^{14}$ :

- Mal-absorption: The development of idiopathic bile acid mal-absorption has been observed following enteric infections, which may be result in diarrhea-predominant IBS.

- Increase in entero-endocrine cells/lymphocytes: An increase in serotonin-containing entero-endocrine cells and $\mathrm{T}$ lymphocytes, has been demonstrated following acute Campylobacter enteritis.

- Antibiotic use: The use of antibiotics for GI or other infections may be a risk factor for developing functional bowel symptoms.

- Bacterial overgrowth- Small intestinal bacterial overgrowth may be associated with IBS ${ }^{15}$.

\subsection{Alteration in Fecal Microflora}

Changes in the composition of intestinal microflora may be associated with IBS. Patients with diarrhea-predominant IBS would benefit from probiotics, which influence the composition and metabolism of the microflora ${ }^{16}$.

\subsection{Food Sensitivity}

The role of food in the pathophysiology of IBS is not clear. Some patients with IBS report worsening of symptoms after eating and perceive food intolerance to certain foods. Food allergy, carbohydrate mal-absorption, and gluten sensitivity may be involved in IBS.

\subsection{Food Allergy}

Eliminating specific foods in patients who have elevated $\operatorname{IgG}$ titers associated with those food antigens may reduce IBS symptoms ${ }^{17}$.

\subsection{Carbohydrate Malabsorption}

Fermentable oligo-, di-, and monosaccharides and polyols (FODMAPs) enter the distal small bowel and colon where they are fermented, leading to symptoms and increased intestinal permeability and possibly inflammation ${ }^{18-20}$. Fructose intolerance has been suggested as a possible form of carbohydrate malabsorption contributing to gastrointestinal symptoms such as flatus, pain, bloating, belching and altered bowel habits.

\subsection{Genetics}

Familial studies suggest a modest contribution of genetics to the development of IBS $^{21}$.

\subsection{Psychosocial dysfunction}

Psychosocial factors influence the expression of IBS. Anxiety, sleep problems, somatic symptoms are risk factors for IBS.

3.0. Types of IBS: Four subtypes of IBS were recognized:

3.1. IBS with constipation (hard or lumpy stools $\geq 25$ 
percent / loose or watery stools $<25$ percent of bowel movements)

3.2. IBS with diarrhea (loose or water stools $\geq 25$ percent / hard or lumpy stools $<5$ percent of bowel movements)

3.3. Mixed IBS (hard or lumpy stools $\geq 25$ percent / loose or watery stools $\geq 25$ percent of bowel movements)

3.4. Un-subtyped IBS (insufficient abnormality of stool consistency to meet the above subtypes)

\subsection{Clinical features}

The clinical features of IBS are lower abdominal cramps, gas and bloating, constipation or diarrhea, may be both, feeling the immediate urgency to have bowel movement when waking up as well as during or after eating, feeling of incomplete emptying even after having defecation, pain relief after defecation, presence of mucus in stools, may have psychological symptoms like anxiety and depression and physical findings are unremarkable.

\subsection{Investigations}

No investigation is usually needed. Investigations are done to exclude the differential diagnosis. Since many disorders present with symptoms similar to IBS, other causes of abdominal pain should be excluded by Complete blood count, ESR \& CRP, Stool for ova, parasites, and blood, Abdominal x-rays, Urinalysis, Serum tTG IgA, MT, CXR, Breath tests for fructose and lactose intolerances, Breath test for small intestine bacterial overgrowth (SIBO), Upper GI endoscopy and Colonoscopy. Routine laboratory studies are normal in IBS.

5.1. Red flags in diagnosing IBS: The red flags in diagnosis of IBS are confirmed weight loss, nocturnal or progressive abdominal pain, unexplained anemia, bleeding per rectum, severe diarrhea and/or vomiting, delayed puberty, unexplained fever and strong family history of inflammatory bowel.

6.0. Treatment of irritable bowel syndrome

Treatments include counseling, dietary management, medication, physical activity and psychotherapy.

6.1. Counseling: The focus of treatment should be on relief of symptoms and addressing the patient's concerns. Patients should be informed of the chronic and benign nature of IBS, and also informed that the diagnosis is not likely to be changed, and that he or she should have a normal life span.

6.2. Dietary Management: A careful dietary history may reveal patterns of symptoms related to specific foods. A number of dietary interventions have been proposed but their efficacy has not been well established ${ }^{22}$.

\subsubsection{Lactose}

Lactose free diet should be considered in patients suspected IBS. Exclusion of gas-producing foods, likesbeans, onions, celery, carrots, raisins, bananas, apricots, prunes, brussels sprouts, wheat germ, pretzels, and bagels should be avoided.

\subsubsection{Food Allergies}

Food allergy may have role in the development of symptom.

\subsubsection{Gluten Sensitivity}

Gluten sensitivity (without overt celiac disease) has been proposed as a cause of functional bowel disorders. 6.2.4. Carbohydrate Mal-absorption

Fermentable oligo-, di-, and monosaccharides and polyols (FODMAPs) in patients with IBS or IBD enter the distal small bowel and colon where they are fermented, leading to symptoms. Some examples of FODMAPs include: Fructans (found in wheat, onions, and artichokes), Galactans (found in legumes, cabbage, and Brussels' sprouts), Lactose, Fructose, Sorbitol, Xylitol, Mannitol

\subsubsection{Fiber}

A decrease in fiber intake to $12 \mathrm{~g}$ per day (particularly insoluble fiber such as bran) was suggested in a British guideline, because of the potential of fiber to exacerbate symptoms. An increase in the intake of fiber is often recommended. Proposed mechanisms for fiber's beneficial effects include: enhancement of water holding properties of the stool; formation of gels to provide lubrication; bulking of the stool; and binding of agents such as bile. Clinical trials found no convincing evidence that the commonly-used bulking agents were more effective than placebo at relieving global IBS symptoms. Although the efficacy of fiber supplements has not been proven ${ }^{23}$, some improvement has been demonstrated in patients with IBS whose primary complaints are abdominal pain and constipation. Dose of fibre is age plus 5 grams of fiber daily.

\subsection{Medication}

Pharmacologic agents are only an adjunct to treatment in irritable bowel syndrome (IBS). Furthermore, the drug chosen varies depending on the patient's major symptoms; diarrhea-predominant IBS is treated differently from constipation-predominant disease.

6.3.1. Antispasmodic agents: The antispasmodic agents include

6.3.1.1. Directly affect intestinal smooth muscle relaxation eg, mebeverine, pinaverine, pepperment oil.

Mebeverine is licensed in the United Kingdom and is generally well tolerated; and can be used on an as required basis before meals. A systematic review ${ }^{24}$ 
which searched medical databases and all relevant literature from 1965 to June 2009 for any placebo-controlled clinical trials of mebeverine, identified 14 relevant papers ( 8 were randomized trials with 555 patients) and concluded that mebeverine is mostly well tolerated with no significant adverse effects; however, its efficacy in global improvement of IBS was not found to be statistically significant ${ }^{24}$.

6.3.1.2. Act via their anticholinergic or antimuscarinic properties like Dicyclomine and hyoscyamine, duration on demand basis; Inhibition of GI smooth muscle reduces stimulated colonic motor activity \& may be beneficial in patient with postprandial abdominal pain, gas, bloating, and fecal urgency.

\subsubsection{Antidepressants}

Antidepressants have analgesic properties independent of their mood improving effects and may therefore be beneficial in patients with neuropathic pain. The postulated mechanisms of pain modulation with tricyclic antidepressants( TCA) and possibly serotonin reuptake inhibitors in IBS are facilitation of endogenous endorphin release, blockade of norepinephrine reuptake leading to enhancement of descending inhibitory pain pathways, and blockade of the pain neuro-modulator, serotonin. TCAs, via their anti-cholinergic properties, also slow intestinal transit time, which provide benefit in diarrhea-predominant IBS. Improvement in neuropathic pain with TCAs occurs at lower doses than required for treatment of depression. As a result, if an antidepressant is chosen for the treatment of IBS, low doses should be administered initially and titrated to pain control or tolerance. Because of the delayed onset of action, three to four weeks of therapy should be attempted before considering treatment insufficient and increasing the dose. Examples of antidepressant medications used in patients with IBS include amitriptyline, imipramine, nortriptyline, and desipramine. The initial dose should be adjusted based upon tolerance and response. TCAs should be used cautiously in patients with constipation. Amitriptyline, nortriptyline, and imipramine can be started at a dose of 10 to $25 \mathrm{mg}$ at bedtime and increased every three to four weeks based upon clinical response and tolerance $\mathrm{e}^{25}$. Desipramine is used in a similar fashion beginning at 12.5 to $25 \mathrm{mg}$ at bedtime. If the patient is intolerant of one TCA, another may be tried. Paroxetine (10 to 20 $\mathrm{mg}$ daily), fluoxetine (20 to $40 \mathrm{mg}$ daily), sertraline (50 to $100 \mathrm{mg}$ daily), or other antidepressant medications can be considered if depression is a cofactor.

\subsubsection{Benzodiazepines}

Anxiolytic agents are of limited usefulness in IBS because of the risk of drug interactions, habituation, and rebound withdrawal. Furthermore, benzodiazepines may lower pain thresholds by stimulating gamma aminobutyric acid (GABA) receptors, thereby decreasing brain serotonin. They may, however, be useful for short-term (less than two weeks) reduction of acute situational anxiety that may be contributing to symptoms.

\subsubsection{5-hydroxytryptamine 3 receptor antagonists}

5-hydroxytryptamine-3 receptor antagonists such as ondansetron, alosetron and graniosetron modulate visceral afferent activity from the gastrointestinal tract and may improve abdominal pain.

\subsubsection{5-hydroxytryptamine 4 receptor agonists}

Agonists of 5-hydroxytryptamine-4 receptor stimulate the release of neurotransmitters and increase colonic motility, providing a rationale for their use in constipation predominant IBS. The first of this class of drugs is tegaserod was approved for IBS and constipation but removed from the market due to cardiovascular side-effects.

\subsubsection{Lubiprostone}

Lubiprostone is a locally acting chloride channel activator that enhances chloride-rich intestinal fluid secretion. Lubiprostone has been approved by the United States Food and Drug Administration for treatment of irritable bowel syndrome with constipation in women 18 years and older. The approved dose is 8 micrograms twice daily for 12 weeks $^{26}$.

\subsubsection{Linaclotide}

An agonist of guanylatecyclase, linaclotide is a unique agent which was recently approved by both American and European regulatory agencies for the treatment of IBS-C. Stimulation of guanylatecyclase receptors leads to increased secretion of both guanylin and uroguanylininto the intestinal lumen where they act as a second messenger for both fluid and electrolyte release into the large bowel ${ }^{27}$. Linaclotide is minimally absorbed and has a strong affinity for the guanylatecyclase receptor.

\subsubsection{Other Drugs}

Pepperment oil, laxatives-polyethylene glycol, mast cell stabilizer, antibiotics-rifaximin, probiotics, loperamide

\subsubsection{Mast cell stabilizers}

Ketotifen is a mast cell stabilizer that has been studied for the treatment of IBS based upon the theory that mast cell activation contributes to visceral hypersensitivity. In a randomized trial of 60 patients, the use of ketotifen for eight weeks increased the threshold for discomfort with rectal distension in patients who were hypersensitive to rectal balloon distension at baseline, but not in those with normal sensitivity at baseline. 
in patients who received ketotifen compared with those who received placebo, the results did not reach statistical significance.

\subsubsection{Antibiotics}

Rifaximin, a nonabsorbable antibiotic, led to symptomatic improvement in global IBS symptoms and bloating. The mechanisms leading to the benefit are unclear but may be due to suppression of gas producing bacteria in the colon. It is reasonable to consider two-week trial of rifaximin ${ }^{28}$.

\subsection{Physical Activity and Psychosocial Therapy}

\subsubsection{Physical activity}

Increased physical activity may help with the symptoms of IBS. Increased physical activity was comprised of 20 to 60 minutes of moderate to vigorous activity three to five days per week.

\subsubsection{Psychosocial therapies}

Behavioral treatments may be considered for motivated patients who associate symptoms with stressors, although their benefits remain controversial. Hypnosis, biofeedback, and psychotherapy help to reduce anxiety levels, encourage health promoting behavior, increase patient responsibility and involvement in the treatment, and improve pain tolerance.

\section{Conclusion}

IBS is a clinical diagnosis of exclusion. The diagnosis is challenging because of the nature and range of associated symptoms and their interpretation amongst parents and pediatricians. A detailed focused history and use of Rome III criteria will be helpful to clarify uncertainties about the diagnosis. Investigations should be kept to the minimum and used for selected cases to exclude other serious pathologies with similar presenting features. For successful management of IBS in children initially enough time should be spent at explaining and reassuring the child and the parents. Therapy needs to be individualized to patient needs. Before initiating therapy it is important to explain the expected benefits and possible side-effects to the family. Multi-disciplinary team approach is needed for management of difficult cases.

\section{References}

1. Drossman DA. The functional gastrointestinal disorders and the Rome III process. Gastroenterology 2006;130:1377-90

2. Munich (Germany): World Gastroenterology Organization (WGO); 2009. World Gastroenterology Organization (WGO). World Gastroenterology Organization Global Guideline: Irritable Bowel Syndrome: A Global Perspective; p. 20

3. Thompson WG, Heaton KW, Smyth GT, Smyth C. Irritable bowel syndrome in general practice: Prevalence, characteristics, and referral. Gut 2000;46:78-82
4. Chang FY, Lu CL, Chen TS. The current prevalence of irritable bowel syndrome in Asia. J Neurogastroenterol Motil 2010; 16: 389-400

5. Makharia GK, Verma AK, Amarchand R, Goswami A, Singh P, Agnihotri A, et al. Prevalence of irritable bowel syndrome: A community based study from northern India. J NeurogastroenterolMotil2011;17:82-7

6. Huerta I, Valdovinos MA, Schmulson M. Irritable bowel syndrome in Mexico. Dig Dis 2001; 19: 251-257

7. Kellow JE, Eckersley GM, Jones M. Enteric and central contributions to intestinal dysmotility in irritable bowel syndrome. Dig Dis Sci 1992;37:168-74

8. Whitehead WE, Palsson OS. Is rectal pain sensitivity a biological marker for irritable bowel syndrome: Psychological influences on pain perception. Gastroenterology 1998;115:1263-71

9. Delvaux M. Role of visceral sensitivity in the pathophysiology of irritable bowel syndrome. Gut 2002;51(Suppl 1):i67-71

10. Kim DY, Camilleri M. Serotonin: A mediator of the brain-gut connection. Am J Gastroenterol 2000;95:2698-709

11. Kellow JE, Eckersley GM, Jones M. Enteric and central contributions to intestinal dysmotility in irritable bowel syndrome. Dig Dis Sci 1992;37:168-74

12. Barbara G, Stanghellini V, De Giorgio R, Cremon C, Cottrell GS, Santini D, et al. Activated mast cells in proximity to colonic nerves correlate with abdominal pain in irritable bowel syndrome. Gastroenterology 2004;126:693-702

13. Chadwick VS, Chen W, Shu D, Paulus B, Bethwaite P, Tie A, et al. Activation of the mucosal immune system in irritable bowel syndrome. Gastroenterology 2002;122:1778-83

14. Barbara G, De Giorgio R, Stanghellini V, Cremon C, Salvioli B, Corinaldesi R. New pathophysiological mechanisms in irritable bowel syndrome. Aliment Pharmacol Ther 2004; 20(2):1-9

15. Khoshini R, Dai SC, Lezcano S, Pimentel M. A systematic review of diagnostic tests for small intestinal bacterial overgrowth. Dig Dis Sci 2008;53:1443-54

16. Swidsinski A, Weber J, Loening-Baucke V, Hale LP, Lochs H. Spatial organization and composition of the mucosal flora in patients with inflammatory bowel disease. J Clin Microbiol 2005; 43: 3380-3389

17. Boettcher E, Crowe SE. Dietary Proteins and Functional Gastrointestinal Disorders. Am J Gastroenterol 2013;108:728-736 18. El-Salhy M. Irritable bowel syndrome: diagnosis and pathogenesis. World J Gastroenterol 2012;18: 5151-5163

19. Marcason W. What is the FODMAP diet? J Acad Nutr Diet 2012; 112:1696

20. Lozinsky AC, Boé C, Palmero R, Fagundes-Neto U. Fructose malabsorption in children with functional digestive disorders. Arq Gastroenterol 2013; 50: 226-230

21. Saito YA. The role of genetics in IBS. Gastroenterol Clin North Am 2011;40:45-67

22. Huertas-Ceballos AA, Logan S, Bennett C, Macarthur C. Dietary interventions for recurrent abdominal pain (RAP) and irritable bowel syndrome (IBS) in childhood. Cochrane Database Syst Rev 2009; (1) CD003019

23. Horvath A, DziechciarzP,Szajewska H. Systematic review of randomized controlled trials: fiber supplements for abdominal pain-related functional gastrointestinal disorders in childhood. Ann Nutr Metab 2012;61:95-101

24. Darvish-Damavandi M, Nikfar S, Abdollahi M. A systematic review of efficacy and tolerability of mebeverine in irritable bowel syndrome. World J Gastroenterol 2010;16:547-553

25. Bahar RJ, Collins BS, Steinmetz B, Ament ME. Double-blind placebo-controlled trial of amitriptyline for the treatment of 
irritable bowel syndrome in adolescents. J Pediatr 2008;152:685-689

26. Chey WD, Drossman DA, Johanson JF, Scott C, Panas RM, Ueno R. Safety and patient outcomes with lubiprostone for up to 52 weeks in patients with irritable bowel syndrome with constipation. Aliment Pharmacol Ther 2012; 35: 587-599

27. Bryant AP, Busby RW, Bartolini WP, Cordero EA, HannigG, Kessler MM, Pierce CM,Solinga RM, Tobin JV, Mahajan- Miklos
S, Cohen MB, Kurtz CB, Currie MG. Linaclotide is a potent and selective guanylatecyclase $\mathrm{C}$ agonist that elicits pharmacological effects locally in the gastrointestinal tract. Life Sci 2010; 86: 760-765

28. Meyrat P, Safroneeva E, Schoepfer AM. Rifaximin treatment for the irritable bowel syndrome with a positive lactulose hydrogen breath test improves symptoms for at least 3 months. Aliment Pharmacol Ther 2012; 36: 1084-1093 